\title{
NEW AND NOTEWORTHY RECORDS OF MOSSES FROM DOI (MT.) INTHANON, CHIANG MAI, CHOM TONG DISTRICT, NORTHERN THAILAND
}

\author{
Narin Printarakul, Benito C. TAN, Kanya Santanachote \\ \& HIROYUKI AKIYAMA
}

\begin{abstract}
Mosses new to Thailand (35 species in 29 genera) and new to Doi Inthanon (6 species in 6 genera) are reported based on collections made by the authors. Austinia tenuinervis var. micholitzii W. R. Buck \& H. A. Crum, Brotherella nictans (Mitt.) Broth., Chionostomum hainanensis B. C. Tan \& Y. Jia, Clastobryopsis muelleri (Dixon) Tixier, Trichosteleum stigmosum Mitt., Micralsopsis complanata (Dixon) W. R. Buck, and Fissidens schwabei Nog. are fully illustrated.
\end{abstract}

Key words: Doi Inthanon, flora, moss, Thailand

Narin Printarakul \& Kanya Santanachote, CMU Herbarium, Department of Biology, Faculty of Science, Chiang Mai University, Chiang Mai 50200, Thailand; e-mail: kame_cobtor@hotmail.com \& ksantanachote@gmail.com

Benito C. Tan, Department of Biological Science, National University of Singapore, Singapore 119260; e-mail: dbsbct@nus. edu.sg

Hiroyuki Akiyama, Museum of Nature and Human Activities, Hyogo, 6-chome, Yayoigaoka, Sanda 669-1546, Japan; e-mail: akiyama@hitohaku.jp

\section{INTRODUCTION}

Doi (Mt.) Inthanon is located in the Doi Inthanon National Park, Chiang Mai Province, northern Thailand. The park, established in 1972 and located $50 \mathrm{~km}$ to the southwest of the city of Chiang Mai, comprises 48, 240 ha and ranges from $400 \mathrm{~m}$ to $2565 \mathrm{~m}$ in altitude. Doi Inthanon has an average annual rainfall of $1908 \mathrm{~mm}$, ranging from 1229 to $2561 \mathrm{~mm}$ over a 7-year period (measured in 1993-1999 at the Royal Project Office of Doi Inthanon National Park at $1300 \mathrm{~m}$ alt.). This montane forest has a typical tropical monsoonal climate with a 5- to 6-month dry season. Rainfall increases with altitude and clouds frequently cover the mountain above $1500 \mathrm{~m}$ alt. even in the dry season (Santisuk 1988) and thus dense mossy forests are developed in Doi Inthanon over $1500 \mathrm{~m}$ alt., especially above $2000 \mathrm{~m}$ alt.

We have been making bryological collections at Doi Inthanon National Park from 2000 to 2011, primarily at four localities, located at $1300 \mathrm{~m}$ alt. (rather dry, hill evergreen forest below the lower limit of cloud zone), $1700 \mathrm{~m}$ alt. (hill evergreen forest above the lower limit of cloud zone, mainly within a 15 -ha permanent monitoring plot), 2250 $2300 \mathrm{~m}$ alt. (open roadside, at the edge and inside mossy montane evergreen forests), and the summit area (2500-2565 m alt., including Ang-ka trail surrounding a small marsh covered by dense mossy forest). In the course of our floristic surveys in these localities, we found a number of mosses new to Thailand or new to Doi Inthanon. The determination of their new record status is based on Akiyama (2006), Nathi et al. (2010), Pollawatn et al. (2008), Printarakul et al. (2012), Tan et al. (2006), and Wongkuna et al. (2009) as well as the following two checklists, He 2005-2013 and Tan \& Iwatsuki (1993). For detailed information of the permanent monitoring plot situated at $1700 \mathrm{~m}$ alt., see Akiyama (2010) and Akiyama et al. (2011).

We use the following symbols: ${ }^{*}-$ new to Doi Inthanon (6 species in 6 genera); ${ }^{* *}$ - new to Thailand (35 species in 29 genera). Additional symbol 
(') means species previously reported without notice in Akiyama et al. (2011) from the permanent plot at $1700 \mathrm{~m}$ alt. All the specimens are deposited at BKF, CMU or HYO. Species are listed alphabetically by generic name with information on altitude of collection sites with names and numbers of the collectors. Three species previously described on separate papers based on the specimens collected from Doi Inthanon by the authors, i.e., Clastobryopsis imbricata H. Akiyama, Ying Chang \& B. C. Tan. (Akiyama et al. 2010), Indopottia irieandoana H. Akiyama (Akiyama \& Goffinet 2011), and Symphyodon leiocarpus H. Akiyama \& Tsubota (Akiyama \& Tsubota 2009) are also included in this list.

\section{LIST OF MOSS SPECIES}

\section{**Atractylocarpus comosus Dixon}

$2500 \mathrm{~m}$ alt., Akiyama et al. 21540.

${ }^{* *}$ Austinia tenuinervis (Mitt.) Müll.Hal. var. micholitzii W. R. Buck \& H. A. Crum

Figs $1 \& 7 \mathrm{c}, \mathrm{d}$ $2300 \mathrm{~m}$ alt., Printarakul 2778 \& 2794, Akiyama et al. 1456 \& 1457, Akiyama \& Printarakul 2778 \& 2794.

NOTE. This species formed thin carpets on tree trunk. It looks like Micralsopsis complanata in branching pattern of ascending stems and ovate leaves with short laminal cells, but differs in well developed costa in leaves, perforation in exostome teeth, and absence of endostome. This species has been known from Borneo and Mindanao (Buck \& Crum 1978; Tan \& Iwatsuki 1991).

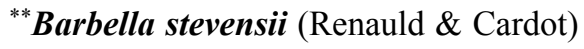

\section{Fleisch.}

$2300 \mathrm{~m}$ alt., Akiyama et al. 1189 \& 1230; $2500 \mathrm{~m}$ alt. (Ang-ka trail), Akiyama et al. 1477 \& 1520.

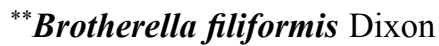

$2300 \mathrm{~m}$ alt., Akiyama et al. 1296.

\section{${ }^{* *}$ Brotherella nictans (Mitt.) Broth.}

Fig. 2

$2300 \mathrm{~m}$ alt., Akiyama et al. 1514.
NoTE. This species is characterized by rather small size for the genus and long acuminate leaves with dense serration.

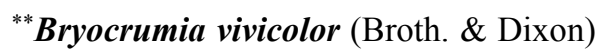

W. R. Buck

Fig. 8c, d

$2300 \mathrm{~m}$ alt., Akiyama et al. 1289.

Note. This species was distinctive in the obtuse leaves arranged in flat foliation along a short prostrating stem. It was found at a single locality in Doi Inthanon where it formed a thin turf on more or less wet boulder at a streamside in a dense montane forest. It has been known from India (Buck 1987).

** Bucklandiella subsecunda (Hook. \& Grev. ex Harv.) Bednarek-Ochyra \& Ochyra Fig. 8e

$2300 \mathrm{~m}$ alt., Akiyama et al. 1155, Printarakul 2788, 2914.

NOTE. This species forms dense, large mats at sunny and open sites on boulders along the summit road.

${ }^{* *}$, Calyptrochaeta ramosa (M. Fleisch.) B. C. Tan \& H. Rob. subsp. ramosa

$1700 \mathrm{~m}$ alt., Akiyama et al. 132, $306 \&$ 407; $2500 \mathrm{~m}$ alt., Akiyama et al. 1531.

${ }^{*}$ Chionostomum hainanensis B. C. Tan \& Y. Jia Fig. 3

$1300 \mathrm{~m}$ alt., Akiyama et al. 1040.

NOTE. Chionostomum hainanense is originally described from China (Hainan Island). It differs from C. rostratum (Griff.) Müll.Hal., previously known from Doi Inthanon, in larger plants size and clearly heterophyllioid alars in stem leaves (Tan \& Jia 1999).

Clastobryopsis imbricata H. Akiyama, Ying Chang \& B. C. Tan.

$1700 \mathrm{~m}$ alt., Akiyama et al. 26-b, 31 (TYPE), 185, 273, 280-b, 287, 358-b, Ando 433.

NOTE. For more details, see Akiyama et al. (2010). 


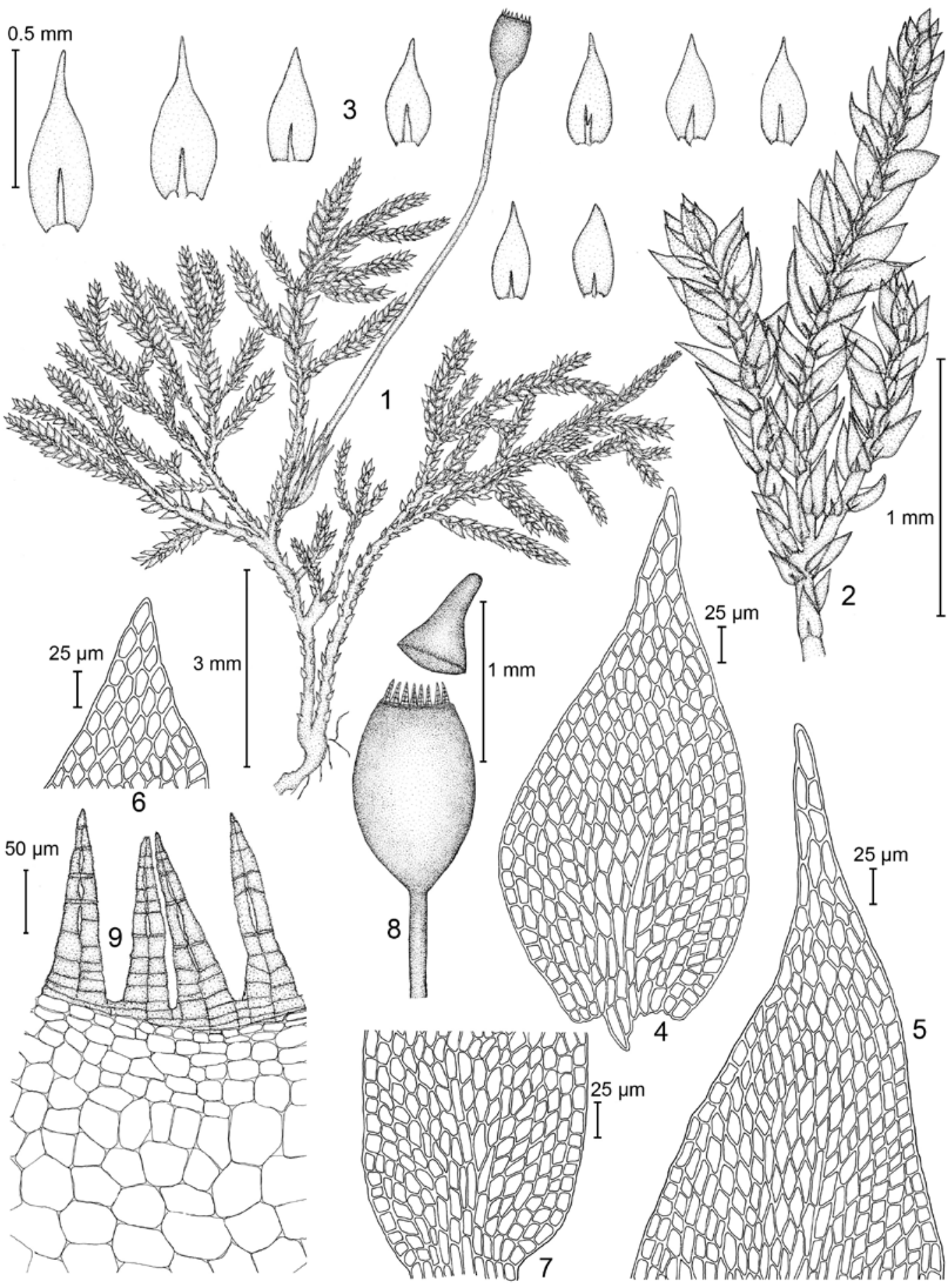

Fig. 1. Austinia tenuinervis (Mitt.) Müll. Hal. var. micholitzii W. R. Buck \& H. A. Crum (Fabroniaceae). 1 - habit with sporophyte, 2 - branches, 3 - leaves, 4 - lamina cells, 5-6 - leaf apex cells, 7 - leaf base cells, 8 - capsule and operculum, 9 - peristome teeth. Drawn from Printarakul 2788 and sporophyte from Printarakul 2794 (CMU). 

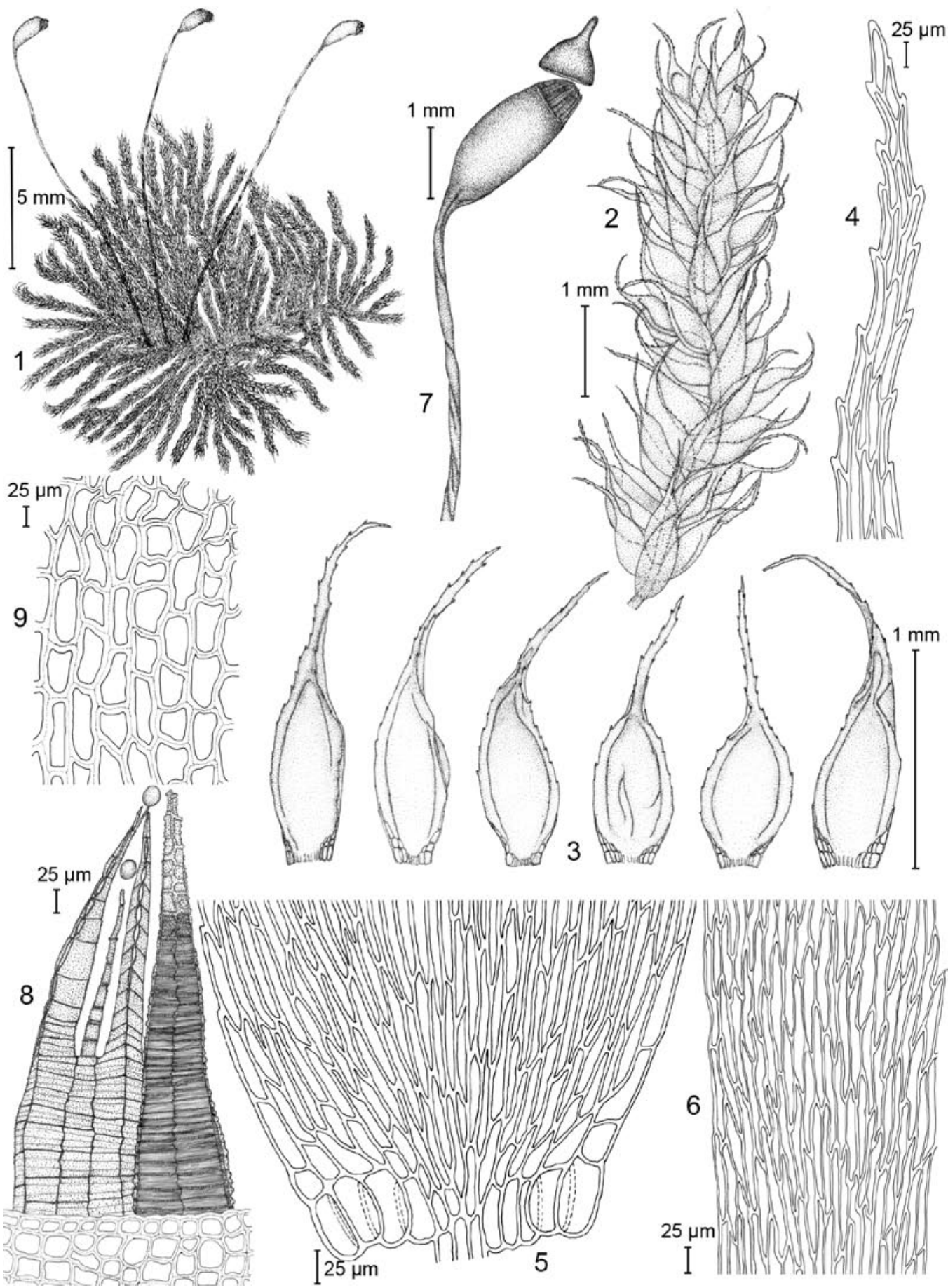

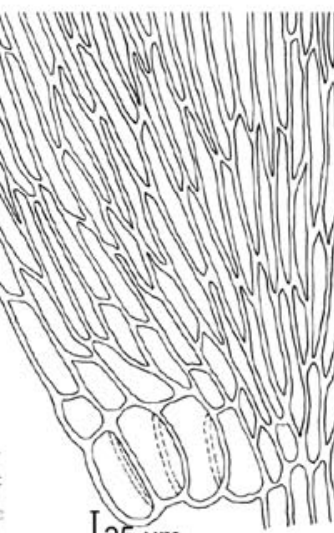

$25 \mu \mathrm{m}$

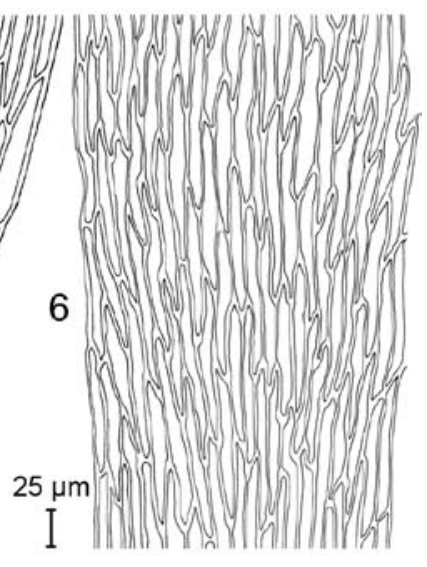

5

Fig. 2. Brotherella nictans (Mitt.) Broth. (Sematophyllaceae). 1 - habit with sporophytes, 2 - branch, 3 - leaves, 4 - leaf apex cells, 5 - leaf base cells, 6 - middle lamina cells, 7 - capsule and operculum, 8 - peristome teeth and spores, 9 - exothecial cells of capsule. Drawn from Printarakul 2808 (CMU). 

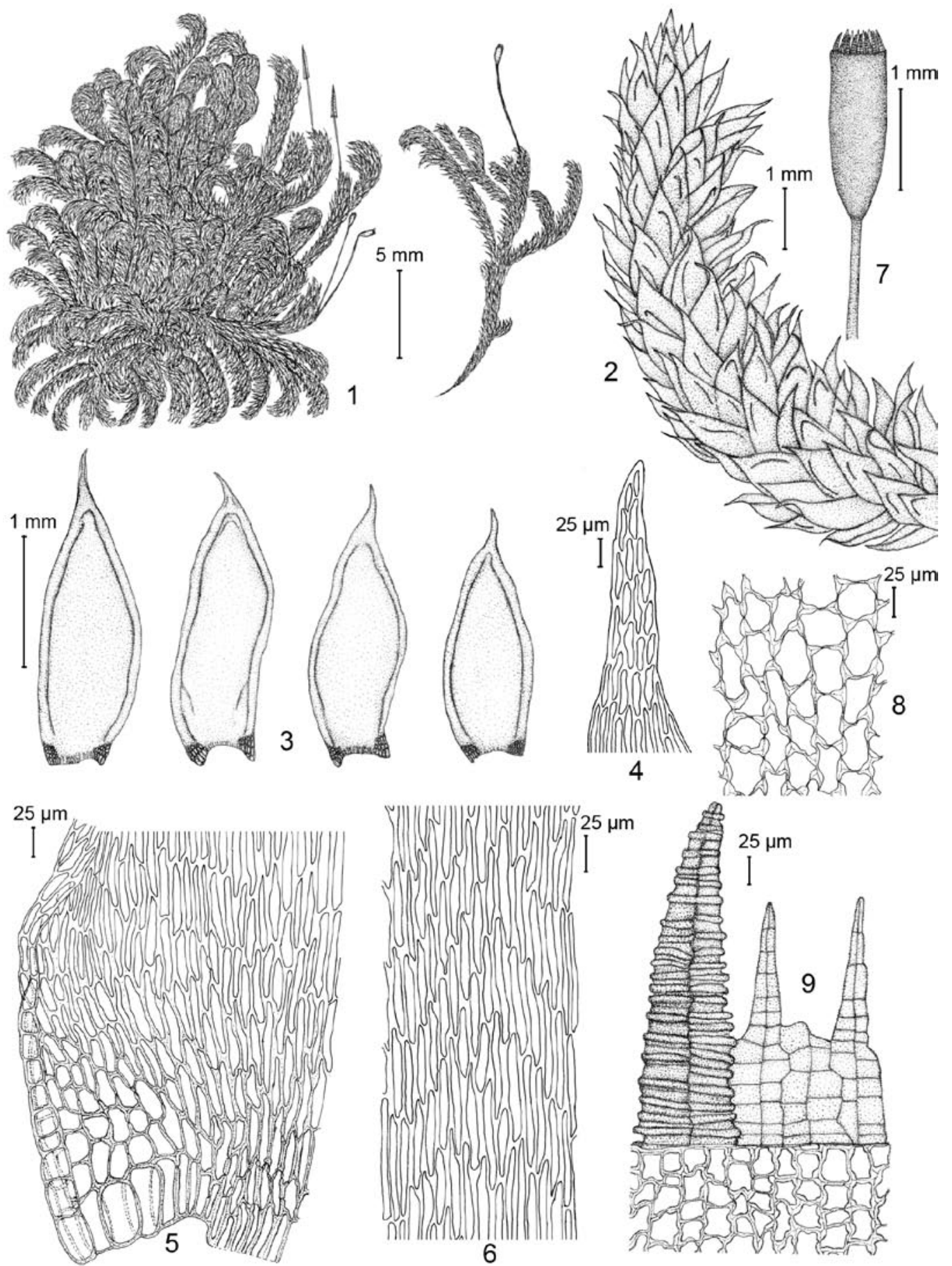

Fig. 3. Chionostomum hainanensis B. C. Tan \& Y. Jia (Sematophyllaceae). 1 - habit with sporophytes, 2 - branch, 3 - leaves, 4 - leaf apex cells, 5 - leaf base cells, 6 - middle lamina cells, 7 - capsule, 8 - exothecial cells of capsule, 9 - peristome teeth. Drawn from Printarakul 842 and sporophyte from Printarakul 2563. (CMU). 


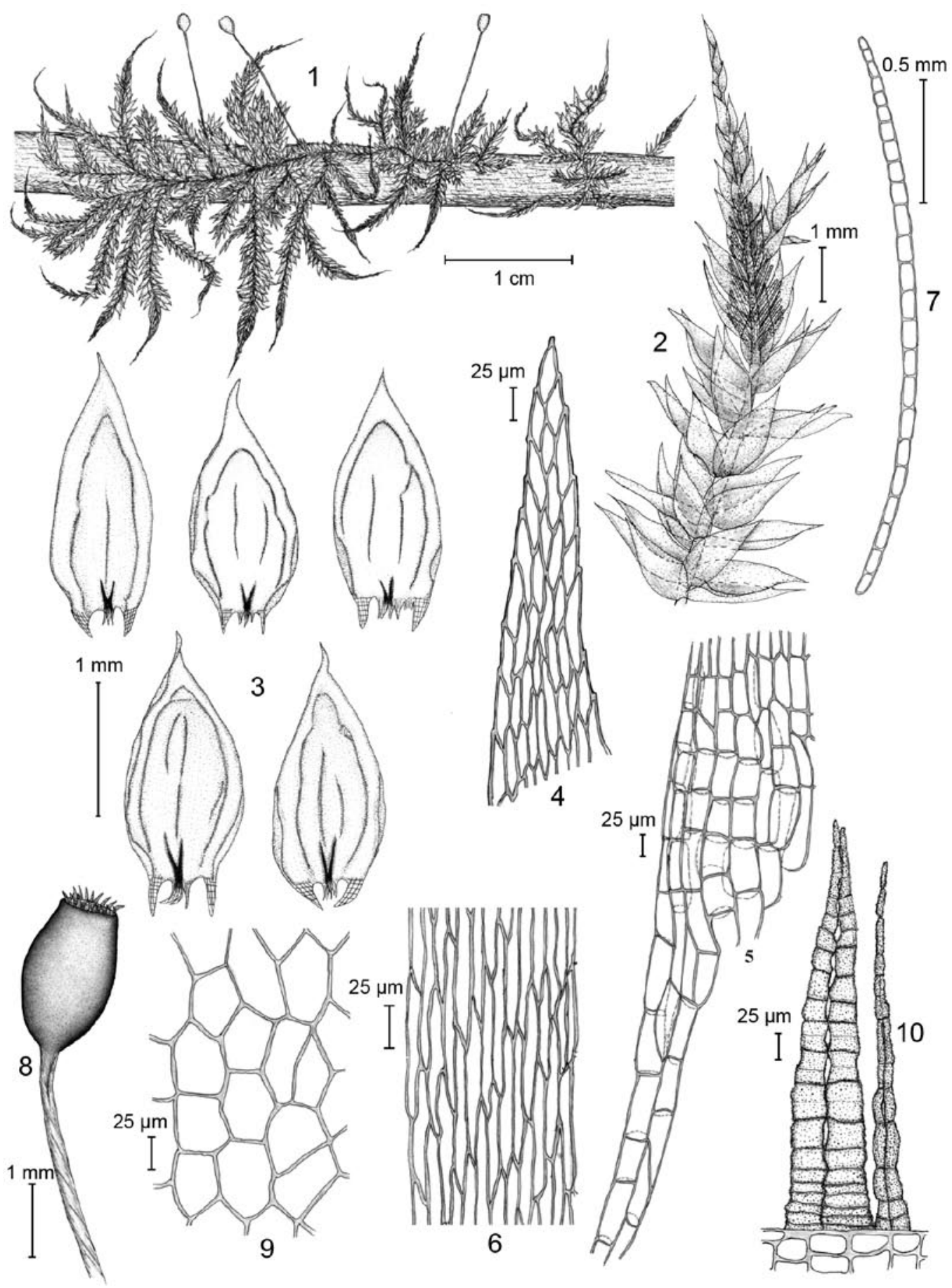

Fig. 4. Clastobryopsis muelleri (Dixon) Tixier (Pylaisiadelphaceae). 1 - habit with sporophytes, 2 - branch, 3 - leaves, 4 - leaf apex cells, 5 - leaf base cells, 6 - middle lamina cells, 7 - filamentous gemma, 8 - capsule, 9 - exothecial cells of capsule, 10 - peristome teeth. Drawn from Printarakul 3027 (CMU). 


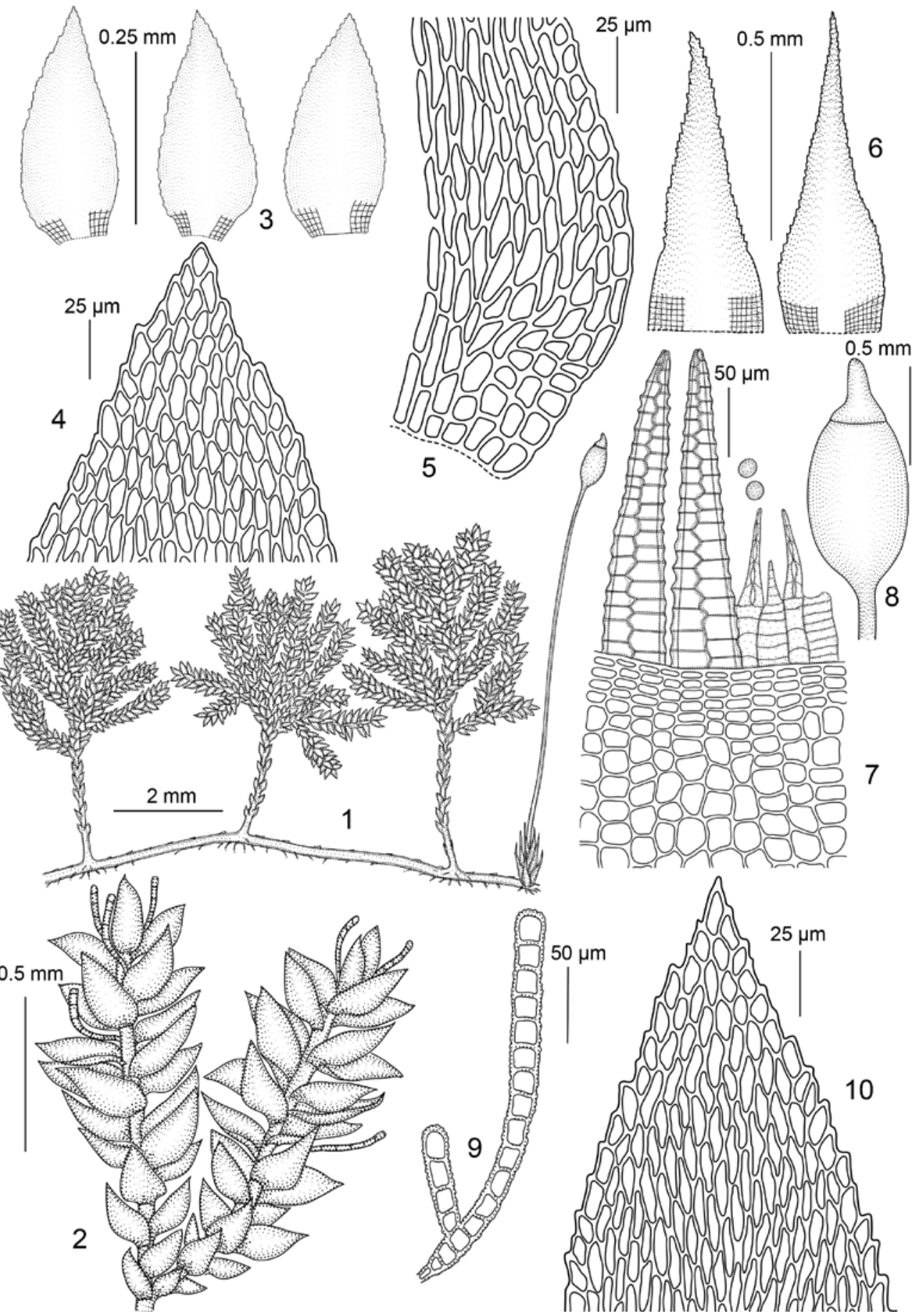

Fig. 5. Micralsopsis complanata (Dixon) W. R. Buck (Fabroniaceae). 1 - habit with sporophyte, 2 - branches, 3 - leaves, $4 \& 10$ - leaf apex cells, 5 - leaf base cells, 6 - perichaetial leaves, 7 - peristome teeth, 8 - capsule and operculum, 9 - gemma. Drawn from Printarakul 3646 (CMU). 


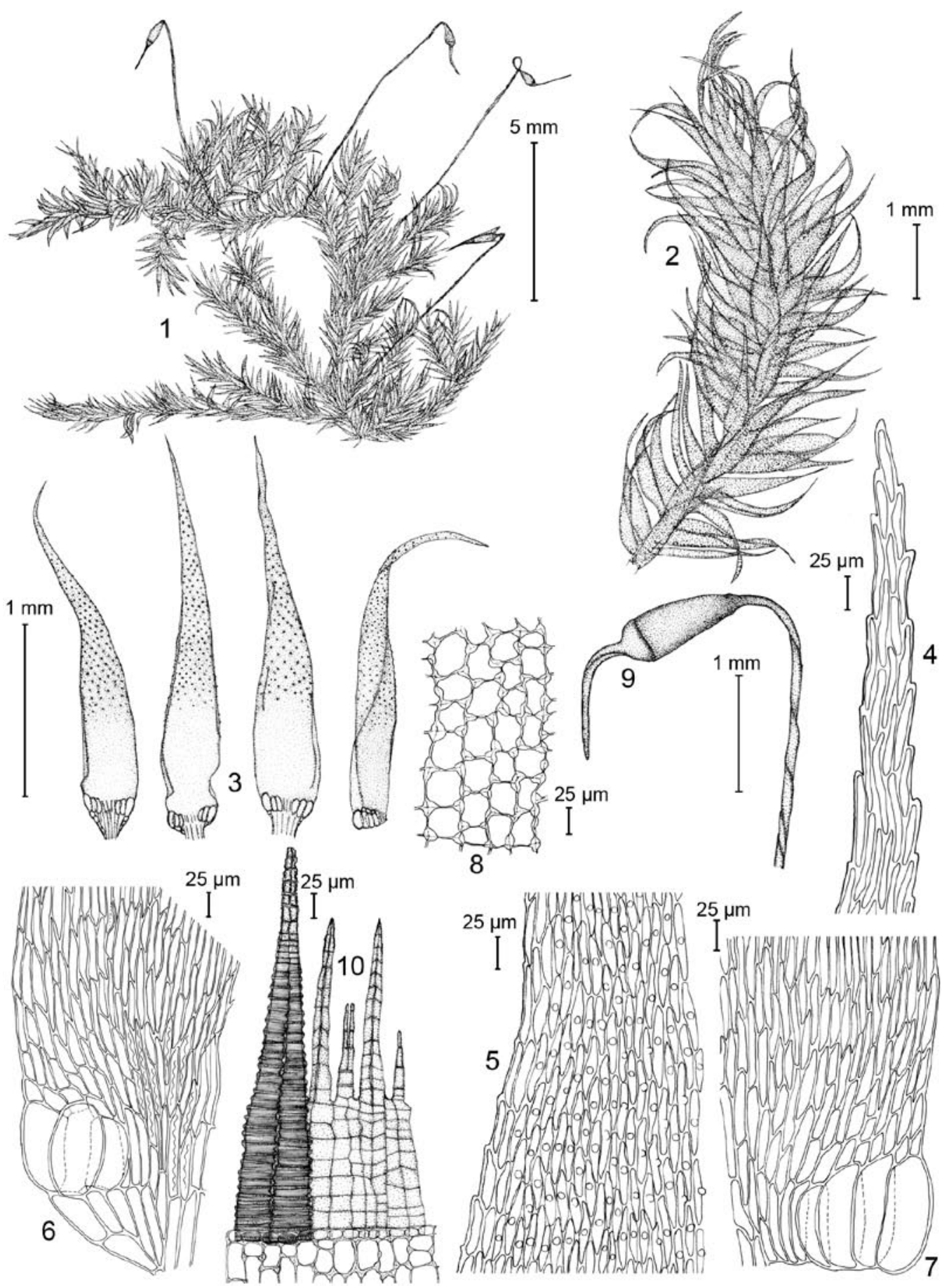

Fig. 6. Trichosteleum stigmosum Mitt. (Sematophyllaceae). 1 - habit with sporophytes, 2 - branch, 3 - leaves, 4 - leaf apex cells, 5 - middle lamina cells, $6 \& 7$ - leaf base cells, 8 - exothecial cells of capsule, 9 - capsule and operculum, 10 - peristome teeth. Drawn from Santanachote 01-01 (CMU). 
${ }^{*}$ Clastobryopsis muelleri (Dixon) Tixier Fig. 4 $2550 \mathrm{~m}$ alt., Akiyama et al. 21542.

Note. Ovate and concave leaves with long decurrent bases are characteristic from the other species of Clastobryopsis found from Doi Inthanon. Printarakul et al. (2012) recently reported this species from Doi Pui, Chiang Mai.

\section{** Daltonia apiculata Mitt.}

2280 m alt., Akiyama et al. 1353.

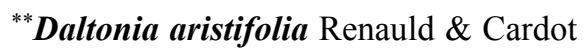

$1700 \mathrm{~m}$ alt., Akiyama et al. 1130 \& 1131.

\section{${ }^{* *}$ Daltonia semitorta Mitt.}

2300 m alt., Akiyama et al. 1536 \& 1555-a, Printarakul 3695; $2500 \mathrm{~m}$ alt., Akiyama et al. 1496.

\section{**, 'Desmatodon leucostoma (R. Br.) Berggr.}

$1700 \mathrm{~m}$ alt., Akiyama et al. 424.

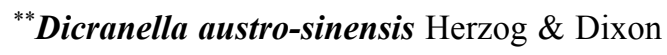
$2300 \mathrm{~m}$ alt., Akiyama et al. 1159.

\section{**, 'Diphyscium mucronifolium Mitt.}

$1700 \mathrm{~m}$ alt., Akiyama et al. 1134; $2250 \mathrm{~m}$ alt., Akiyama et al. 1288.

\section{**, !Ditrichum laxissimum (Mitt.) Kuntze}

$1700 \mathrm{~m}$ alt., Ando s.n.

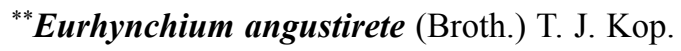

$2300 \mathrm{~m}$ alt., Akiyama et al. 1297.

${ }^{* *}$ Eurhynchium savatieri Schimp. ex Broth. $2300 \mathrm{~m}$ alt., Akiyama et al. 1305 \& 1310.

**, Eurhynchium hians (Hedw.) Sande Lac.

$1700 \mathrm{~m}$ alt., Akiyama et al. 68, 94, 238 \& 397.

${ }^{* *}$ Fissidens schwabei Nog. $\quad$ Fig. 7a, b

$1650 \mathrm{~m}$ alt., Akiyama et al. 1140, Printarakul 2639.
NOTE. This species has small plants with sporophytes. It grows on shrub twigs and resembles Fissidens guandongensis Z. Iwats. \& Z. H. Li. in its outer morphology. The type specimen (Schwabe $66, \mathrm{NICH}$ ) was collected in Taiwan (http://hattoribot-lab.com/species/Fissidens-schwabei.pdf).

${ }^{* *}$, ! Glossadelphus lingulatus (Cardot) M. Fleisch. $1700 \mathrm{~m}$ alt., Akiyama et al. 369, 380 \& 396.

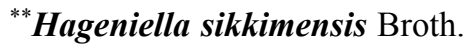
Fig. 7e, $\mathrm{f}$ $1300 \mathrm{~m}$ alt., Akiyama et al. 1401; $2300 \mathrm{~m}$ alt., Akiyama et al. $1094,1317 \& 1328$.

NOTE. This species forms thin, large mats on the root of trees.

\section{**,!Hookeriopsis geminidens Broth.}

$1700 \mathrm{~m}$ alt., Akiyama et al. 178.

\section{Indopottia irieandoana $\mathrm{H}$. Akiyama}

$1700 \mathrm{~m}$ alt., Akiyama et al. 422 (TYPE) \& 426.

Note. For more details, see Akiyama and Goffinet (2011).

\section{**,!Leucoloma amoene-virens Mitt.}

$1700 \mathrm{~m}$ alt., Akiyama et al. 160, 303 \& 382 .

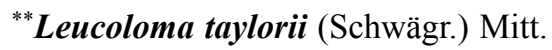

$1300 \mathrm{~m}$ alt., Akiyama et al. 1029 \& 1436-a.

***acrothamnium leptohymenioides Nog.

$2300 \mathrm{~m}$ alt., Akiyama et al. 1221; $2500 \mathrm{~m}$ alt. (Angka trail), Akiyama et al. 1486.

**, Micralsopsis complanata (Dixon) W. R. Buck Figs $5 \& 8 \mathrm{a}, \mathrm{b}$

$1700 \mathrm{~m}$ alt., Akiyama et al. 26-a, 121, 148, 193, 201, 251, 307, 387, 406, $1128 \& 1137 ; 2300 \mathrm{~m}$ alt., Printarakul 3646, Akiyama et al. 1013, 1016, 1064, 1084, 1090, 1093, 1128, 1137, 1176, 1191, 1207, 1279\& 1329, Akiyama \& Printarakul 1434 \& 1458.

NOTE. The species forms thin and sparse turfs on tree trunk and branches and not uncommon. 

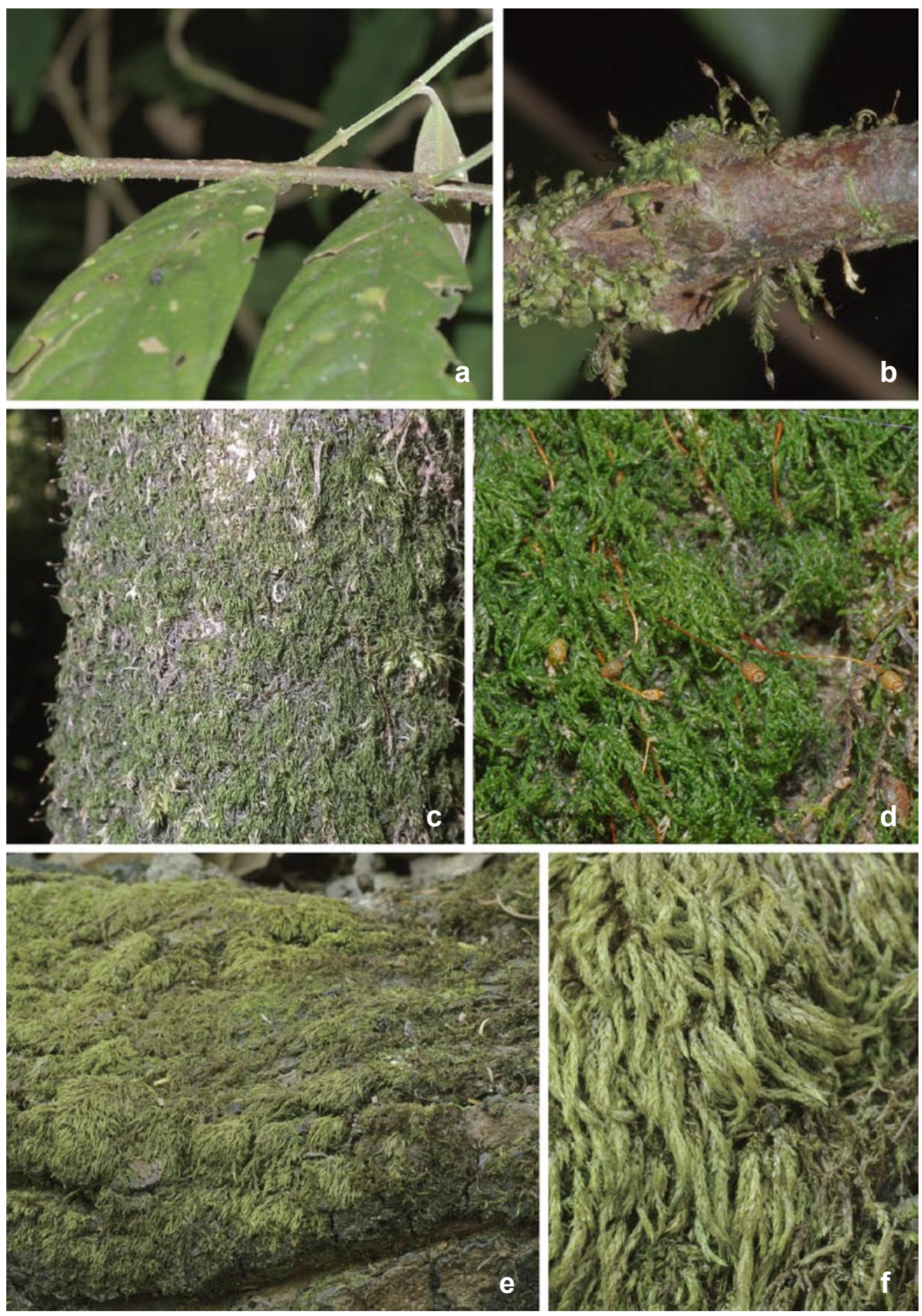

Fig. 7. a \& b - Fissidens schwabei Nog. (Doi Inthanon, $1650 \mathrm{~m}$ alt.). $\mathrm{c} \& \mathrm{~d}$ - Austinia tenuinervis var. micholitzii (Broth. ex Dixon) W. R. Buck \& H. A. Crum (Doi Inthanon, $2300 \mathrm{~m}$ alt.). e \& f - Hageniella sikkimensis Broth. (Doi Inthanon, $1300 \mathrm{~m}$ alt.). 

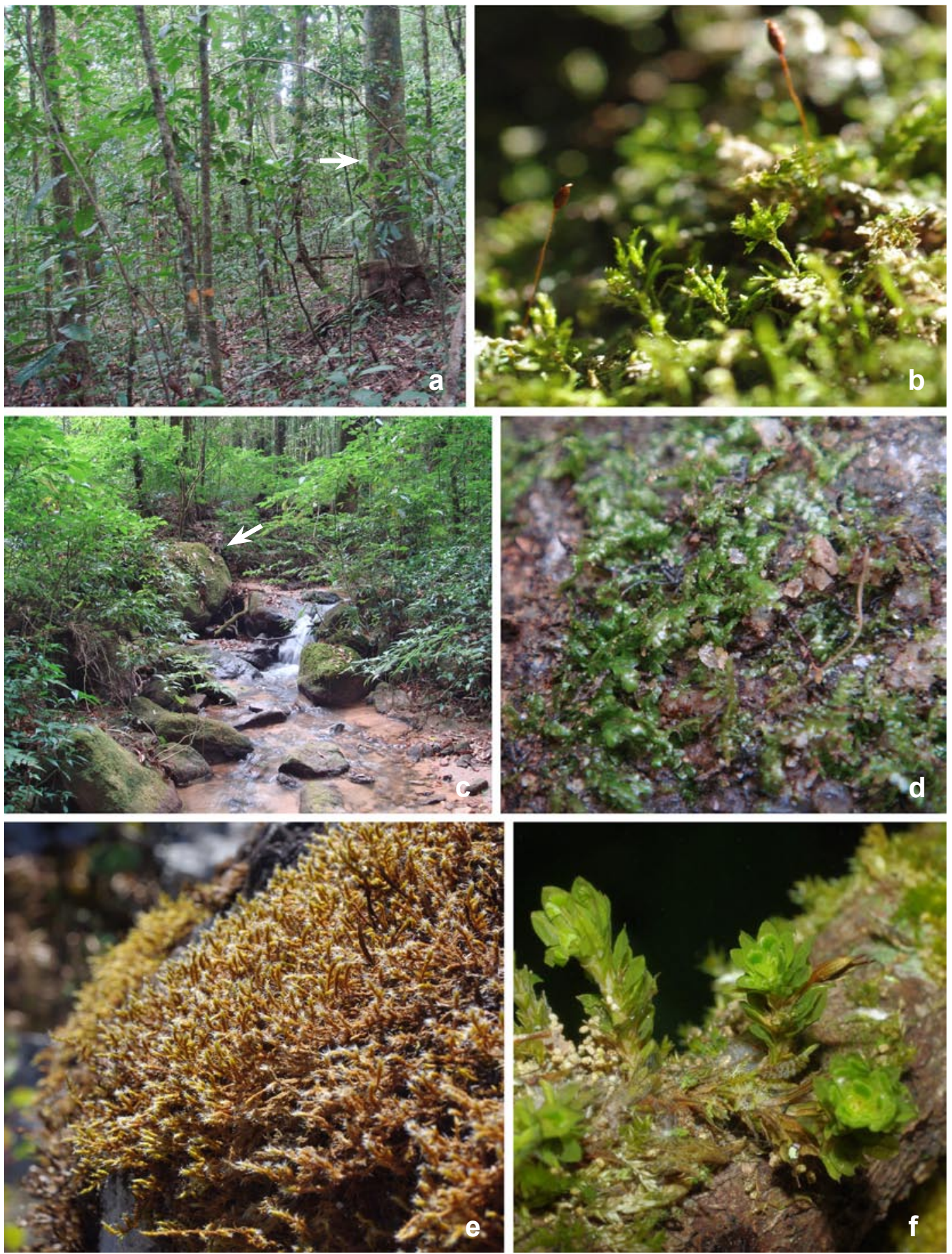

Fig. 8. a \& b, Micralsopsis complanata (Dixon) W. R. Buck (Doi Inthanon, $2300 \mathrm{~m}$ alt.). c \& d - Bryocrumia vivicolor (Broth. \& Dixon) W. R. Buck (Doi Inthanon, $2300 \mathrm{~m}$ alt.). e - Bucklandiella subsecunda (Hook. \& Grev. ex Harv.) Bednarek-Ochyra \& Ochyra (Doi Inthanon, $2300 \mathrm{~m}$ alt.). f - Syrrhopodon semiliber (Mitt.) Besch. (Doi Inthanon, $1700 \mathrm{~m}$ alt.). 
Ascending stems showing pinnately branching pattern with sporophytes are distinctive. Buck (1987) reported this interesting moss from India (Assam) and China (Yunnan).

**, !Papillaria chrysoclada (Müll.Hal.) A. Jaeger

$1700 \mathrm{~m}$ alt., Akiyama et al. 114, 158, \& 223.

${ }^{* *}$ Pelekium haplohymenium (Harv. \& Hook.f.) A. Touw

$1700 \mathrm{~m}$ alt., Akiyama et al. 1001, $1003 \& 1225 ; 2300 \mathrm{~m}$ alt., Akiyama et al. 1182.

**, !Philonotis mollis (Dozy \& Molk.) Mitt.

$1700 \mathrm{~m}$ alt., Akiyama et al. $412 \& 416$.

\section{*Pinnatella amblyphylla Enroth}

$1700 \mathrm{~m}$ alt., Akiyama et al. 1489, Printarakul 2677, $2688, \& 3672$; second report of this species from Thailand (Enroth 1994).

NoTE. The type specimen of this Thailand endemic species is from Doi Chiang Dao (Chiang Mai District). This species formed small patches at the base of tree trunks on the forest floor. We found another 'species' slightly different from the typical $P$. amblyphylla in leaf morphology and occupying a different habitat (on wet boulder at streambed) at a single locality of $1700 \mathrm{~m}$ alt.

\section{*** Pogonatum nudiusculum Mitt.}

$2250 \mathrm{~m}$ alt., Akiyama 1315 \& 1330.

NOTE. See Eddy (1988: 39), who gave Thailand as the distribution area of this species without referring to any voucher specimens.

\section{*Pohlia flexuosa Hook.}

$2300 \mathrm{~m}$ alt., Akiyama et al. 1170 \& 1557.

NotE. Printarakul et al. (2012) recently reported this species from Doi Suthep and Doi Pui, Chiang Mai District.

${ }^{* *}$ Pohlia proligera (Kindb.) S. O. Lindberg ex Arnell

$2300 \mathrm{~m}$ alt., Akiyama et al. 1165.
Symphyodon leiocarpus H. Akiyama \& Tsubota

$1700 \mathrm{~m}$ alt., Akiyama et al. 314 \& 332; $2550 \mathrm{~m}$ alt., Akiyama Th-51 (TYPE), Th-127, Th-128 and many others. (2009)

Note. For details, see Akiyama and Tsubota

*, !Syrrhopodon semiliber (Mitt.) Besch. Fig. $8 \mathrm{f}$

$1700 \mathrm{~m}$ alt., Akiyama et al. 18, 195, 285, 288, 300, $312 \& 316$.

NOTE. Dense and greenish filamentous gemmae produced on the upper half of leaves are very distinctive for Syrrhopodon semiliber. It has been known from central and southern parts of Thailand (He 2005-2013).

**, ! Thuidium pseudoglaucinum A. Touw

$1700 \mathrm{~m}$ alt., Akiyama et al. 106, $357 \& 1146$.

${ }^{*}$ Trichosteleum stigmosum Mitt.

Fig. 6

$1300 \mathrm{~m}$ alt., Akiyama et al. 1409; $2300 \mathrm{~m}$ alt., Akiyama et al. $1086 \& 1098$.

NOTE. This species grows on decayed logs in rather dry, lower montane forests. Printarakul et al. (2012) recently reported this species from Doi Pui, Chiang Mai District.

**, !Warburgiella leptorhynchoides (Mitt.) M. Fleisch.

$1700 \mathrm{~m}$ alt., Ando s.n.

${ }^{* *}$ Weisiopsis anomala (Broth. \& Paris) Broth. \& Paris

$1700 \mathrm{~m}$ alt. Akiyama et al. 1427.

${ }^{* *}$ Wijkia deflexifolia (Mitt. ex Renauld \& Cardot) H.A.Crum

$2500 \mathrm{~m}$ alt., Akiyama 21537-a.

TAXA EXCLUDED FROM THAILAND FLORA

Distichophyllum decolyi Gangulee

This species was earlier reported from Thailand by Akiyama (2006), but the specimen is referable to 
the widely distributed and highly variable $D$. maibarae Besch (Ho et al. 2010, p. 114).

\section{Distichophyllum obovatum (Griff.) Paris}

This species was earlier reported from Thailand by Akiyama (2006), but the specimen is referable to D. wanianum B. C. Tan \& P. J. Lin. (Ho et al. 2010, p. 120).

ACKNOWLEDGMENTS. We thank to National Research Council of Thailand for their kind permission of collecting activities in Doi Inthanon National Park (NRCT, Registration No. 83/52), Dr. Johannes Enroth (University of Helsinki) for confirming the identity of Pinnatella amblyphylla, Professor Ryszard Ochyra for identifying Bucklandiella subsecunda, and Professor Rodney Seppelt (Tasmanian herbarium) for correction of English text. This study was financially supported by CHE-Ph. D. Scholarship (CHE500163, 2007-2012) and the grant from the Graduate School of Chiang Mai University (2007-2012) to NP, JSPS KAKENHI Grant Numbers (24570108) to HA, and JSPS KAKENHI (20405008 and 24255007) to Dr. M. Kanzaki (Kyoto University).

\section{REFERENCES}

AKIYAMA H. 2006. New records of mosses from Thailand. Trop. Bryol. 28: 59.

AKIYAMA H. 2010. Scopelophila cataractae found growing on tree trunks in northern Thailand. Trop. Bryol. 32: 97-99.

AKIYAMA H. \& GOFFINET B. 2011. Indopottia irieandoana sp. nov. (Pottiaceae) from Doi Inthanon, northern Thailand. J. Bryol. 33(2): 122-129.

AkiYama H. \& Tsubota H. 2009. Symphyodon leiocarpus, sp. nov. (Symphyodontaceae, Musci) from Thailand, classified in the new subgenus Macrothamniopsis. Acta Phytotax. Geobot. 60(2): 87-96.

AKIYAma H., Chang Y. \& TAN B. C. 2010. Clastobryopsis imbricata (Pylaisiadelphaceae) sp. nov. from Doi Inthanon, northern Thailand. Bryologist 113(4): 752-759.

AKIYAMA H., FURUKI T., SRI-NGERNYUANG K. \& KANZAKI M. 2011. Alphabetical list of bryophytes occurring in a 15 ha long-term monitoring plot at Doi Inthanon, Northern Thailand. Bryol. Res. 10 (6): 153-164.
BUCK W. R. 1987. Notes on Asian Hypnaceae and associated taxa. Mem. New York Bot. Gard. 45: 519-527.

BuCK W. R. \& CRUM H. A. 1978. A re-interpretation of the Fabroniaceae with notes on selected genera. J. Hattori Bot. Lab. 44: 347-369.

EDDY A. 1988. Handbook of Malesian Mosses. 1. Sphagnales to Dicranales. British Museum (Natural History), London.

EnRoth J. 1994. A taxonomic monograph of the genus Pinnatella (Neckeraceae, Bryopsida). Acta Bot. Fenn. 151: $1-90$.

HE S. 2005-2013. An annoted checklist and atlas of the mosses of Thailand. [15 March 2013]. http://www.mobot.org./ MOBOT/moss/Thailand/checklist.sthml.

Ho B. C., TAN B. C. \& NATHI Y. 2010. New and noteworthy records of Distichophyllum (Daltoniaceae, Bryopsida) and allied genera in Asia and Australasia. Trop. Bryol. 31: $106-122$.

NATHi Y., TAN B. C. \& SeElanan T. 2010. Ten new records of mosses from Doi Inthanon National Park in Thailand. Gard. Bull. Singapore 61(2): 389-400.

Pollawatn R., Frahm J. P. \& BoOnKerd T. 2008. New species records of Sematophyllaceae (Musci) from Thailand, pp. 41-48. In: Mohamed H., BAKer B. B., Boyce A. N. \& LEE P. K. Y. (eds) Bryology in the new millennium. University of Malaya, Kuala Lumpur.

Printarakul N., TAN B. C., SANDANAchote K. \& WongKUNA K. 2012. Nine new records of mosses from Doi Suthep-Pui National Park and a new variety of Fissidens from Thailand. Cryptog. Bryol. 33(1): 23-31.

SANTISUK T. 1988. An account of the vegetation of Northern Thailand. Franz Steiner Verlag Wiesbaden, Stuttgart.

TAN B. C. \& Iwatsuki Z. 1991. A new annotated Philippine moss checklist. Harvard Pap. Bot. 3: 1-64.

TAN B. C. \& IWATSUKI Z. 1993. A new checklist of Indochinese mosses. J. Hattori Bot. Lab. 74: 325-405.

TAN B. C. \& JIA Y. 1999. A preliminary revision of Chinese Sematophyllaceae. J. Hattori Bot. Lab. 86: 1-70.

TAN B. C., Ho B.-C., Linis V., IsKandar E. A. P., NurhaSANAH I., DAMAYANTi L., MUlyati SRI \& HAERIDA I. 2006. Mosses of Gunung Halimun National Park, West Java, Indonesia. Reinwardtia 12: 205-214.

Wongkuna K., Santanachote K. \& Tan B. C. 2009. Miscellaneous observation on Fissidens in Thailand with five new species records. Cryptog. Bryol. 30(2): 301-309. 